\title{
QUOTIENT OF A LOOP GROUP AND WITTEN GENUS
}

\author{
Rémi Léandre
}

\section{INTRODUCTION}

Let us consider the free loop space $L(M)$ of a compact manifold: it is endowed with a natural circle action. The fixed point set of this circle action is the manifold himself.

In finite dimension, when there is a circle action over a manifold, there are two types of relations between the fixed point set and the total space. It is the purpose of the Berline-Vergne localization formulas ([B.V]) and of the Lefschetz formulas $\left(\left[\mathrm{Bi}_{2}\right],[\mathrm{T}]\right)$. In the first case, we localize the integrals over the fixed point set; in the second case, using the method of Taubes $([\mathrm{T}])$, we localize the operators over the fixed point set.

Over the loop space, the Berline-Vergne localization formulas give the index theorem for the Dirac operator over a manifold $\left(\left[\mathrm{Bi}_{3}\right],\left[\mathrm{Bi}_{4}\right],[\mathrm{At}]\right)$. If we localize an operator, this gives the Index theorem over the loop space, which gives quantities which are very important for algebraic topology as it was noticed by Witten ([Wi], [Se], [H.B.J]).

The problem for Lefschetz formula over the loop space is that it is purely hypothetical. Taubes ([T]) gives a rigorous construction for a limit model. He considers as model of the free loop space the family of flat loops on the tangent space. He considers a measure over the flat loop space, which lives over random distributions, and whose choice comes from quantum field theory. So we cannot curve its model.

There is another way to attack the problem of defining the Dirac operator over the loop space and others geometrical operators: it is the way followed by Léandre-Roan ([L.R]), Jones-Léandre ([J.L]) and Léandre $\left(\left[\mathrm{L}_{3}\right]\right.$ ) (See in $\left[\mathrm{L}_{7}\right]$ the survey of Léandre ). We consider the Brownian bridge measure. There is a tangent Hilbert space, which allows to state integration by parts formulas over the free loop space. [L.R] meets the problem that the tangent Hilbert space is not stable by Lie brackets; a connection allows to compute a regularized stochastic exterior derivative and to compute its adjoint. This gives a rigorous approach to the Euler-Poincaré characteristic of Dixon-Harvey-Vafa-Witten of an orbifold ([D.H.V.W]). $\left[\mathrm{L}_{3}\right]$ meets the same problem for the Dolbeault operator over the loop space of a Kaehler manifold, with the natural polarization over the loop space which results from the polarization of the Kaehler manifold. This gives a rigorous approach to Hirzebruch's genus of level N $([\mathrm{H}])$.

The problem is to construct the Dirac operator over the loop space. [J.L] present only a beginning of construction of it. Namely, if we follow Taubes approach to construct the Dirac operator over the free loop space, we have to extend over the free loop space the natural polarization which arises from Fourier series. This leads to complicated considerations.

We remark that over a loop group, if we consider the tangent space of a loop group, we don't meet the previous difficulties. For instance, the tangent space of a loop group is stable by Lie bracket. This allows to compute a true exterior derivative and its adjoint (and not a regularized one as in [J.L] and [L.R]) in [F.Fr]. The polarization by Fourier series is well defined over the loop group. But the Witten genus of a group is trivial, because the tangent bundle of a Lie group $G$ is trivial.

The idea is to consider $(L G) / H$ where $H$ is a maximal torus of $G$ where $G$ is a non commutative Lie group. It inherites clearly a circle action over it, and its fixed point set coincides with a countable disjoint unions of the homogeneous space space $G / H$

So the natural conjecture is the following:

Conjecture (Bismut): Over $(L G) / H$, there is a Dirac-Taubes operator which is invariant under the natural circle action over $(L G) / H$ and it is possible to define its "renormalized" equivariant Index which is is equal to the Witten genus of $G / H$.

The purpose of this work is to give a stochastic approach to this conjecture.

We construct in the first part an $H$-equivariant operator over $L G$, which generalizes the construction of Taubes. For that, we suppose that the homogeneous space $G / H$ is Kaehlerian and spin. This operator is invariant under the natural circle action over $L G$. The space of functionals over $(L G) / H$ can be seen 
as a functional over $L G$ which is invariant under the $H$ action, which induces an action by isometries over it. Since $H$ is finite dimensional, the analysis over $(L G) / H$ comes ultimately to the analysis over $L G$ for $H$ invariant functionals. This allows to define a Dirac operator over $(L G) / H$, which follows precisely the prescriptions of Taubes.

In a second time, we consider the loop group in small time, and we repeat the considerations in this loop group context of $[\mathrm{L} . \mathrm{R}],[\mathrm{J} . \mathrm{L}]$ and $\left[\mathrm{L}_{3}\right]$ to motivate the conjecture. We deform the Hilbert space of the theory and we arrive at the limit to a limit operator at the manner of Taubes, whose equivariant index is the Witten genus $G / H$. The price to pay in order to be able to do the asymptotic expansion of the Dirac-Taubes operator near the constant loops is that we have to change completely the structure of the considered Dirac operator. In particular we suppose $G /$ spin and Kaehlerian, and we work in order to perform Bismut's dilatation over the small elements of $L G / H$. The reader interested by short time asymptotic expansion can see the surveys of Kusuoka $([\mathrm{K}])$, Watanabe $([\mathrm{Wa}])$ and Léandre $\left(\left[\mathrm{L}_{1}\right]\right)$

The question which is put by this work is the following: Taubes construction of the Dirac operator over the loop space is a construction by hand, which avoids the theory of spin representation of loop group. On the other hand, there is a theory of spinor fields over the loop space ([C.M], [C.P], [M.L]), and in particular, by using the fact that there is a measure over the loop space, the definition of Hilbert spaces of sections of the spin bundle over the loop space $\left(\left[\mathrm{L}_{4}\right],\left[\mathrm{L}_{5}\right],\left[\mathrm{L}_{6}\right]\right)$ where the Dirac operator should act. What is the relation between the two point of view?

We thank J.M. Bismut for the illuminating suggestion which is at the basis of this work, as well as J.L. Clerc and O. Hijazi..

\section{CONSTRUCTION OF THE DIRAC-TAUBES OPERATOR}

Let $G$ be a compact simply connected Lie group. We consider the Killing metric over its Lie algebra LieG. We consider the Brownian motion over $G$ staring from $g$. It is the solution of the following stochastic differential equation starting from $g$ :

$$
d g_{s}=g_{s} d B_{s}
$$

where $B_{s}$ is a Brownian motion over Lie $G$ and where $d$ denotes the Stratonovitch differential. It has an heat kernel $p_{t}\left(g, g^{\prime}\right)$ with respect to the Haar measure $d \pi(g)$ over $G$. Over the free loop group of $G$ called $L(G)$ of continuous loops, we consider the following measure

$$
d \mu_{G, 1}=\frac{p_{1}(g, g) d \pi(g) \otimes d P_{1, g}}{\int p_{1}(g, g) d g}
$$

where $P_{1, g}$ is the law of the Brownian bridge starting from $g$ and arriving un $g$ in time 1 . For a cylindrical functional $F\left(g_{s_{1}}, . ., g_{s_{r}}\right), s_{1}<s_{2}<. .<s_{r}$, we have a more explicit description of $\mu_{G, 1}$ in terms of the heat kernel:

$$
\begin{gathered}
E\left[F\left(g_{s_{1}}, . ., g_{s_{r}}\right)\right]=\frac{1}{\int p_{1}(g, g) d \pi(g)} \int . . \int p_{s_{2}-s_{1}}\left(g_{1}, g_{2}\right) \\
p_{s_{3}-s_{2}}\left(g_{2}, g_{3}\right) . . p_{s_{r}-s_{1}}\left(g_{r}, g_{1}\right) F\left(g_{1}, . ., g_{r}\right) d \pi\left(g_{1}\right) . . d \pi\left(g_{r}\right)
\end{gathered}
$$

Let us remark that by the symmetry of the equation $(2.1), p_{1}(g, g)=p_{1}(e, e)$ such that $\int p_{1}(g, g) d \pi(g)=$ $p_{1}(e, e)$.

We consider as tangent space of an element $g$. of the loop loop group the set of $X$. where $X_{s}=g_{s} K_{s}$ such that $K_{0}=K_{1}$ and where the path $K$. in the Lie algebra of $G$ is of finite energy. We introduce the following Hilbert structure:

$$
\|X .\|_{g .}=\int_{0}^{1}\left|K_{s}\right|^{2} d s+\int_{0}^{1}\left|K_{s}^{\prime}\right|^{2} d s
$$


There is a natural circle action over $L(G)$ : if $q=\exp [2 i \pi t]$ belongs to the circle, $q(g \text {. })_{s}=g_{s-t}$.

This natural circle action preserves the measure as well the tangent bundle of the loop group: it preserves therefore the metric, since $g$ induce a rotation from $\operatorname{Lie}(G)$ into the tangent space of $G$ at $g$. If we complify the tangent space of $g ; T_{g}$. into $T_{g} \otimes C$ and if the real complex strucure (2.4) is transformed into the natural complex Hilbert structure over the complexified tangent space, there is a natural orthonormal basis of $T_{g} \otimes C$ starting from a real orthonormal basis $e_{i}$ of $\operatorname{Lie}(G)$. It is given by

$$
X_{n}\left(e_{i}\right)_{s}=g_{s} \frac{\exp ^{[2 \pi i n s]} e_{i}}{\sqrt{C n^{2}+1}}
$$

This splitts $T_{g} \otimes C$ into $H_{-}+E+H_{+}$. $H_{+}$corresponds to a strictly positive n, $H_{-}$corresponds to $n>0$ and $E$ to $n=0$. We have therefore a polarization over the loop group which comes from the Fourier expansion.

We remark that the tangent bundle is stable by Lie bracket. If $X_{s}=g_{s} K_{s}(X)$ and $Y_{s}=g_{s} K_{s}(Y)$ where $K_{s}(X)$ and $K_{s}(Y)$ are deterministic, we have

$$
[X, Y]_{s}=g_{s}\left[K_{s}(X), K_{s}(Y)\right]
$$

Moreover, $H_{+}$and $H_{-}$are stable by Lie bracket. Under the natural circle action by $q$ in the circle, $X_{n}\left(e_{i}\right)$. behaves as $q^{-n}$ if $e_{i}$ is deterministic.

There is a Levi-Civita connection over $L(G)$ which is given for real vector fieds $X$., $Y$. and $Z$. by a formula of $[\mathrm{K} . \mathrm{N}] \mathrm{p} 160$. We have:

$$
\begin{aligned}
2 & <\nabla_{X_{.}} Y_{.}, Z_{.}>=X_{.}<Y_{.}, Z_{.}>+Y_{.}<X_{.}, Z_{.}>-Z_{.}<X_{.}, Y_{.}> \\
& +<\left[X_{.}, Y_{.}\right], Z .>+<\left[Z_{.}, X_{.}\right], Y_{.}>+<X_{.},\left[Z_{.}, Y_{.}\right]>
\end{aligned}
$$

If we consider the vector fields $X_{n}\left(e_{i}\right)$. for $e_{i}$ determinisitic, $<X_{n}\left(e_{i}\right), X_{m}\left(e_{j}\right)>=\delta_{n, m} \delta_{i, j}$ and

$$
<\nabla_{X_{p}(e),} X_{n}(f), X_{m}\left(f^{\prime}\right),>=C\left(p, n, m, e, f, f^{\prime}\right)
$$

is deterministic. Moreover, (2.7) show, if $n$ and $\mathrm{m}$ are positive, that $\nabla_{X_{n}\left(e_{i}\right)} X_{m}\left(e_{j}\right)$. is a linear combination of $X_{n+m}\left(e_{k}\right)$.

The problem is to understand $E$ now.

For that we introduce a compact Lie group $H$ which is a maximal torus of $G$. We suppose that $G / H$ is spin and Kaehlerian. We suppose that the Kaehler structure is compatible with the action of $G$ over $G / H$. We can decompose the complexified Lie algebra of $G$ into $n_{+}+\operatorname{Lie}(H)+n_{-}$. Let us detail how we proceed. Let us introduce the canonical projection $p: G \rightarrow G / H$. $G$ becomes a fibration over $G / H$ with fiver $H$. Let us consider $g \in p^{-1}(x)$ where $x$ denoted the typical element of $G / H$. At $g$, the complexified tangent space of $G, T_{g} \otimes C$, has the direct orthogonal decomposition $n_{+}(g)+T_{p^{-1}(x)} \otimes C+n_{-}(g)$. The fact that that we have a Kaehler structure over $G / H$ implies that $\left[h, f_{+}\right]$belongs still in $n_{+}$if $h$ belongs to the Lie algebra of $H$ and if $f_{+}$belongs to $n_{+}$. The analogous statement remains true for $n_{-}$. By the action of $G, n_{+}(g)$ can be identified with the set of $T^{1,0}$ vectors $\frac{\partial}{\partial z}$ over $G / H$ and $n_{-}(g)$ can be identified with the sets of $T^{0,1}$ vectors $\frac{\partial}{\partial \bar{z}}$ over $G / H$ (Let us recall that $T^{1,0}$ is the set of eigenvectors $\frac{\partial}{\partial z}$ associated to the eigenvalue $i$ for the complex structure $J$ over $G / H$ and that $T^{0,1}$ is the set of eigenvectors $\frac{\partial}{\partial \bar{z}}$ associated to the eigenvalue $-i$ for the complex structure $J$ ).

We deduce a decomposition of $E$ into $N_{+}+O+N_{-}$which is orthogonal. Moreover, this decomposition is invariant by rotation, because the action of $g \in G$ preserves the Kaehler structure of $G / H$.

Let us recall that over $G / H$, there is a $\bar{\partial}$ operator: $T^{1,0}$ forms are called $d z$ and $T^{0,1}$ forms are called $d \bar{z}$. We introduce the Levi-Civita connection over $G / H$ : it preserves, since $G / H$ is supposed Kaehlerian, the dedomposition of the complexified tangent space in $T^{1,0}$ and in $T^{0,1}$. the same is true for the forms. We consider the bundle $\Lambda\left(T^{0,1}\right)$ and we consider the $\bar{\partial}$ operator:

$$
\bar{\partial}_{f}=\sum d \bar{z}_{i} \wedge \nabla_{\frac{\partial}{\partial \bar{z}_{i}}}
$$

for the levi-Civita connection (See [Gi]) where we take the sum over a local orthonormal basis of $T^{0,1}$. We can tensorize this operator by an auxiliary complex hermitian bundle $\xi$ endowed with an Hermitian connection. 
This allows us to construct the Dirac operator over $G / H$ in terms of a twisted Dolbeault operator, if we suppose $G / H$ spin (See [Gi] p186). We consider a square root of the canonical bundle $\Lambda^{n, 0}$, whose curvature is given by $2 R$, which is a closed $4 \pi i Z$ valued form over $G / H$. We consider its dual $\xi^{\prime}$, which can be endowed with a Hermitian structure and a connection whose curvature is $-R$. We consider the $\bar{\partial}$ operator twisted by $\xi^{\prime}$. This gives a realisation of the Dirac operator over $G / H$.

Let $-R$ be the curvature of $\xi^{\prime}$. We construct a complex line bundle over $L(G)$ by proceeding as follows; we consider the form $-p^{*} R$ over $G$ : it is $2 \pi i Z$ valued. We construct the form $-\int_{0}^{1} p^{*}(R)\left(g_{s}\right)() d$.$s over L(G)$. It is still closed $2 i \pi Z$ valued. This form determines, since $G$ is supposed simply connected and therefore

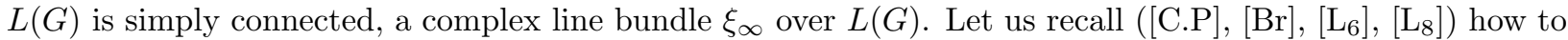
do it, order to construct it. In order to simplify the exposure, we will work over the Holderian loop space $L_{1 / 2-\epsilon}(G)$ : it is possible to assume this because the Brownian loop is almost surely Hoelderian:

$$
\sup \frac{d\left(g_{s}, g_{t}\right)}{d(s, t)^{1 / 2-\epsilon}}<\infty
$$

where $d\left(g_{s}, g_{t}\right)$ denoted the Riemannian distance over the group between $g_{s}$ and $g_{t}$ and $d(s, t)$ denoted the Riemannian over the circle between $s$ and $t$. This will allow us to produce smooth sections of the line bundle $\xi_{\infty}$, whose existence is strongly based upon the existence of partition of unity over the loop space, which does not exist if the loops $g$. are only continuous.

We consider the system of balls $B\left(g_{\alpha} ; \delta\right)$ for the uniform distance of radius $\delta$ and of center the smooth loop $g_{\alpha}$. We construct over this ball the curve

$$
l_{\alpha}\left(g_{.}\right)(t)_{s}=\exp \left[t\left(g_{s}-g_{\alpha, s}\right)\right]
$$

where we consider the exponential of the Lie group. If $g$. belongs to the intersection of $B\left(g_{\alpha}\right.$.; $\left.\delta\right)$ and of $B\left(g_{\beta} ; \delta\right)$, we can construct a system of transition functionals as follows: we join the two loops $l_{\alpha}(g$. $)(t)$. and $l_{\beta}\left(g_{\text {. }}\right)(t)$. by using the Lie group exponential. This constitutes a small triangle. We use the fact that $\Pi_{2}(G)=0$ in order to find a big triangle whose boundary is any path joining $e$. to $g_{\alpha}$., the exponential curve joining $g_{\alpha}$. to $g_{\beta}$. and any curve joinning $g_{\beta}$. to $e$. We produced a distinguished surface $S_{\alpha, \beta}(g$.) in $L_{1 / 2-\epsilon}(G)$ whose boundary is the distinguished path $l_{\alpha}(G)$ joinning $e$. to $g$. and the path $l_{\beta}(g$. $)$ circled in the opposite sense.

We put:

$$
\rho_{\alpha, \beta}(g .)=\exp \left[-2 i \pi \int_{S_{\alpha, \beta}(g .)} \sigma\right]
$$

It is a smooth functional in the traditional sense over the intersection of the two ball $B\left(g_{\alpha .} ; \delta\right)$ and $B\left(g_{\beta} ; \delta\right)$ (and not in the generalized sense of the infinite dimensional analysis). We get clearly, since $\sigma$ is $Z$-valued, over $B\left(g_{\alpha_{1},} ; \delta\right) \cap B\left(g_{\alpha_{2}} ; \delta\right) \cap B\left(g_{\alpha_{3}} ; \delta\right)$

$$
\rho_{\alpha_{1}, \alpha_{2}}\left(g_{.}\right) \rho_{\alpha_{2}, \alpha_{3}}\left(g_{.}\right) \rho_{\alpha_{3}, \alpha_{1}}\left(g_{.}\right)=1
$$

This determines a complex line bundle endowed with a natural Hermition structure over it.

We define an Hermitian connection form over the small ball $B\left(g_{\alpha} ; \delta\right)$ :

$$
A_{\alpha}\left(g_{.}\right)(X .)=\int_{0}^{1} \sigma\left(d / d t l_{\alpha}\left(g_{.}\right)(t), D_{X} l_{\alpha}\left(g_{.}\right)(t)\right)
$$

Let us remark that this Calculus does not need all the schochastic apparatus of $\left[\mathrm{L}_{4}\right],\left[\mathrm{L}_{5}\right]$, [ $\left.\mathrm{L}_{6}\right]$ and $\left[\mathrm{L}_{8}\right]$, because the quantities are here surely defined. The next lemma uses deeply that there exist a partition of unity over $L_{1 / 2-\epsilon}(G)$ associated to the cover $B\left(g_{\alpha} ; \delta\right)$ (See $\left[\mathrm{L}_{8}\right]$ ):

Lemma II.1 There exists a smooth section $\tau_{\xi_{\infty}}$ of $\xi_{\infty}$ such that for all $m>1$

$$
\sum_{n, i} E\left[\left|\nabla_{X_{n}\left(e_{i}\right) .} \tau_{\xi_{\infty}}\right|^{2 m}\right]<\infty
$$


Proof: We choose the section over $B\left(g_{\alpha} ; \delta\right)$ which corresponds to any cylindrical functional in the trivialization given for $A_{\alpha}(g$.). It is not smooth. We multiply it by a mollifer: it is possible, because we consider the Hoelderian loop space. We follow $\left[\mathrm{L}_{8}\right]$. Let us introduce a smooth function $f$ from $[0, \infty]$ into $[1, \infty]$ which is exactly exactly on $\left[0, \delta^{\prime}\right]$ for $\delta^{\prime}<\delta$. We suppose that $f$ behaves as $\left|\delta^{\prime \prime}-r\right|^{-n}$ for a big integer $n$ when $r \rightarrow \delta "$ - for some $\delta "<\delta$ and is infinite for $r>\delta$ ". We introduce a smooth function $h$ from [1, $\infty$ [ into $[0,1]$ with compact suppost which is equal to 1 only at 1 . We consider the functional:

$$
F_{\alpha}\left(g_{.}\right)=h\left(\int_{0}^{1} f\left(d\left(g_{s}, g_{\alpha, s}\right)\right) d s\right)
$$

$F_{\alpha}$ is smooth with support included in $B\left(g_{\alpha} ; \delta\right)$, because we work over the Hoelderian loop space (This property is wrong over the continuous loop space). Moreover:

$$
X_{n}\left(e_{i}\right) . F_{\alpha}\left(g_{.}\right)=h^{\prime}\left(\int_{0}^{1} f\left(d\left(g_{s}, g_{\alpha, s}\right)\right) d s\right) \int_{0}^{1} f^{\prime}\left(d\left(g_{s}, g_{\alpha, s}\right)<d^{\prime}\left(g_{s}, g_{\alpha, s}\right), X_{n}\left(e_{i}\right)_{s}>d s\right.
$$

We get:

$$
\begin{gathered}
\left|X_{n}\left(e_{i}\right) . F_{\alpha}\left(g_{.}\right)\right|^{2} \leq C\left(h^{\prime}\left(\int_{0}^{1} f\left(d\left(g_{s}, g_{\alpha, s}\right)\right) d s\right)^{2}\right. \\
\int_{0}^{1}\left|f^{\prime}\left(d\left(g_{s}, g_{\alpha, s}\right)\right)\right|^{2} d s \int_{0}^{1}\left|<d^{\prime}\left(g_{s}, g_{\alpha, s}\right), X_{n}\left(e_{i}\right)_{s}>\right|^{2} 1_{B\left(g_{\alpha, s} ; \delta\right)}\left(g_{s}\right) d s
\end{gathered}
$$

The product of the two first term in the right hand side of (2.18) is bounded in all the $L^{p}$ by the exponential inequality. The last term has a bound in $n$ and $i$ in $1 /\left(c n^{2}+1\right)$. $\diamond$

Over $L_{1 / 2-\epsilon}(G)$, we can consider the following natural action of $H: g . \rightarrow g . h$. This preserves the measure, the tangent space as well as the Hilbert space structure over the Hilbert space. It commutes with the natural circle action over the loop group. Since $\sigma$ is invariant under the action of $H$ over the loop group, the action of $H$ over the loop group lifts to an action of $H$ over $\xi_{\infty}$, which preserves the Hermitian structure and the connection. It transforms clearly a smooth section of $\xi_{\infty}$ into a smooth section (in the traditional sense). Moreover, if $\tau_{\xi_{\infty}}$ satifies to $(2.16), \tau_{\xi_{\infty}} \cdot h$ satisfies still to $(2.15)$. We can produce an $H$ invariant section by using $\int_{H} \tau_{\xi_{\infty}} . h d \pi(h)$. The space of $H$-invariant sections and of finite linear combinations of $H$-invariant sections produced by Lemma II.1 is dense in the space of $L^{2} H$-invariant section of $\xi_{\infty}$.

This allows to construct the Hilbert space of the theory: we consider $\Lambda\left(H_{-}\right)$the Fermionic Fock bundle of the bundle $H_{-}$and $\Lambda\left(N_{-}\right)$the space of forms associated to the bundle spanned by $X_{0}\left(f_{i}\right)$., $f_{i}$. being a orthonormal basis of $n_{-}$. We consider the bundle $\Xi=\Lambda\left(H_{-}\right) \otimes \Lambda\left(N_{-}\right) \otimes \xi_{\infty}$. It is an Hermitian bundle over the Holderian loop group, endowed with a product connection. Over $\Lambda\left(H_{-}\right)$, we consider the lift to the Levi-Civita connection defined by (2.7). Over $\Lambda\left(N_{-}\right)$, we consider the connection given by $\nabla . X_{0}\left(f_{j}\right)$. $=0$ if $f_{j}$ is a deterministic orthonormal basis of $n_{-}$. This connection is clearly invariant by rotation. Over $\xi_{\infty}$, we consider the connection which is invariant under the $H$-action. The $H$-action over the Hoelderian loop group lift to $\Xi$. More precisely a vector $X_{n}\left(e_{i}\right)$. is trtansformed under the $H$-action into a vector $X_{n}\left(h^{-1} e_{i} h\right)$. This lift keeps the metric and is compatible with the connection which are considered.

Moreover the natural circle action over the loop groups lifts to $\left.\Lambda_{(} H_{-}\right)$, and the connection and the Hermitian metric are compatible with this lift, because the Levi-Civita connection is invariant under the circle action, because the circle acts by isometries. The same considerations work for $\Lambda\left(N_{-}\right)$and for $\xi_{\infty}$, because the form $\sigma$ which determines $\xi_{\infty}$ is invariant under the circle action.

The Hilbert space $E_{H}[\Xi]$ of the theory is the space of sections $\tau_{\Xi}$ of $\Xi$ which are invariant under the $H$-action a and which are in $L^{2}$ : this means that $E\left[\left|\tau_{\Xi}\right|^{2}\right]<\infty$.

We will define a dense core $\Lambda_{H}[\Xi]$, where we can define a regularized Dirac-Taubes operator, which is symmetric, therefore closable.

Let us introduce $I=\left(\left(n_{1}, i_{1}\right), . .,\left(n_{r}, i_{r}\right)\right)$ for $n_{i}<0$ and let us consider $X_{I .}=X_{n_{1}}\left(e_{i_{1}}\right)$. $\wedge . . X_{n_{r}}\left(e_{i_{r}}\right)$. Let $f_{j}$ a given deterministic orthonormal basis of $n_{-}$. Let $J$ be equal to $\left(j_{1}, . ., j_{r}\right)$ and let $Y_{J}$. be equal to 
$X_{0}\left(f_{j_{1}}\right) . \wedge . . \wedge X_{j_{0}}\left(f_{j_{r}}\right) \ldots X_{I}$. and $Y_{J}$. are $H$-invariant, since $e_{j}$ and $f_{j}$ are supposed deterministics. Let $F_{I, J, K}(g$. $)$ be a cylindrical function and let $\tau_{\xi_{\infty}, K}$ a finite family of sections of $\tau_{\xi_{\infty}}$ satisfying (2.15).

A typical section $\tau_{\Xi}$ of the core $\Lambda_{H}[\Xi]$ is a section which is a finite sum of the type:

$$
\tau_{\Xi}=\sum F_{I, J, K}\left(g_{.}\right) X_{I .} \wedge Y_{J} \otimes \tau_{\xi_{\infty}, K}
$$

where we afterwards average under the $H$-action in order to get $H$-invariant sections of $\Xi$. Moreover, it is a dense subspace of the Hilbert space $E_{H}[\Xi]$. We remark that $\Lambda_{H}[\Xi]$ is invariant under the natural circle action over the loop group. It is clearly true for $X_{I}$. and $Y_{J}$. and for the cylindrical functioanls $F_{I, J, K}(g$.) whose set is invariant under the circle action. Ther result arises for $\tau_{\xi_{\infty}, K}$ because the space of sections $\tau_{\xi_{\infty}}$ satisfying $(2.15)$ is invariant under the circle action.

We consider as operator:

$$
\bar{\partial}_{r, \infty}=\sum_{n>0, i} X_{n}\left(e_{i}\right) \wedge \nabla_{X_{n}\left(e_{i}\right) .}+\sum_{f_{j}} X_{0}\left(f_{j}\right) \wedge \nabla_{X_{0}\left(f_{j}\right)} .
$$

$X_{n}\left(e_{i}\right)$. corresponds to the form $Y . \rightarrow<Y, X_{n}\left(e_{i}\right)$. $>$ in the wedge product. By antilinearity of the Hermitian product, it behaves as $q^{n}$ under the action of $q \in S_{1}$, if it is considered as a form. Unfortunately, $\bar{\partial}_{r, \infty}$ is not well defined over $\Lambda_{H}[\Xi]$. In order to see that, we have to estimate $\nabla_{X_{m}\left(e_{i}\right)} X_{k}\left(e_{j}\right)$. when $m \rightarrow \infty$. Let us denote by $\bar{X}$. the natural conjugation of a vector field $X$. which arises from Fourier series. Let us suppose that $X ., Y$. and $Z$. are 3 vector fields of the type $X_{n}\left(e_{k}\right)$. with $e_{k}$ deterministic. By (2.7), we have:

$$
2<\nabla_{X} Y_{.}, Z .>=<\left[X_{.}, Y_{.}\right], Z .>+<Y_{.},\left[Z_{.}, \bar{X} .\right]>+<X_{.},\left[Z_{.}, \bar{Y} .\right]>
$$

We deduce that

$$
\nabla_{X_{m}\left(e_{i}\right) .} X_{l}\left(e_{j}\right) .=\sum_{k} \alpha_{k}(i, j) X_{m+l}\left(e_{k}\right) \cdot \frac{\sqrt{C(m+l)^{2}+1}}{\sqrt{C\left(m^{2}\right)+1} \sqrt{C l^{2}+1}}
$$

for $m>0$ and $l>0$. Therefore $\bar{\partial}_{r, \infty} \tau_{\Xi}$ has absolutely no reason to belong to $E_{H}[\Xi]$, if $\tau_{\Xi}$ belongs to $\Lambda_{H}[\Xi]$.

In order to overcome this difficulty, we will change of connection. We choose

$$
\nabla \cdot X_{m}\left(e_{i}\right) .=0
$$

This connection is clearly invariant by rotation because the vector field $X_{n}\left(e_{i}\right)$. considered as a vector field behaves as $q^{-n}$ under the natural action of the element $q$ of the circle. Moreover this connection is clearly invariant under the $H$-action, because a vector $X_{n}\left(e_{i}\right)$. is trtansformes under the $H$-action in a vector $X_{n}\left(h^{-} e_{i} h\right)$. Therefore, if we change in the definition (2.19) of $\bar{\partial}_{r, \infty}$ of connection following the last prescriptions, $\bar{\partial}_{r, \infty}\left(\tau_{\Xi}\right)$ belongs to $E_{H}[\Xi]$ as soon as $\tau_{\Xi}$ belongs to $E_{H}[\Xi]$ because $X_{m}\left(e_{i}\right) . F_{I, J, K}(g$.$) as a$ behaviour in $\frac{C}{\sqrt{C m^{2}+1}}$ when $m \rightarrow \infty$ and because (2.15)

Let us recall that over the Loop group, we have some integration by parts formulas called the AlbévérioHoegh-Krohn integration by parts formulas ([A.H-K], $\left[\right.$ A.M $\left.\left.\mathrm{M}_{1}\right]\right)$. There exists a functional $\operatorname{div}\left(X_{n}\left(e_{i}\right)\right.$.) which belongs to all the $L^{p}$ over the loop group such that for all cylindrical functional $F$ :

$$
E\left[X_{n}\left(e_{i}\right) . F\right]=E\left[F \operatorname{div}\left(X_{n}\left(e_{i}\right) .\right)\right]
$$

We deduce, since the connection is $\nabla$ is hermitian over $\Xi$, that we can compute the formal adjoint of $\bar{\partial}_{r, \infty}$ called $\bar{\partial}_{r, \infty}^{*}$ :

$$
\bar{\partial}_{r, \infty}^{*}=\sum_{n<0, i}\left(-\nabla_{X_{n}\left(e_{i}\right)} i_{X_{-n}\left(e_{i}\right) .}+\operatorname{div} X_{n}\left(e_{i}\right) \cdot i_{X_{-n}\left(e_{i}\right)}\right)+\sum_{j}\left(-\nabla_{X_{0}\left(f_{j}^{\prime}\right) .} i_{X_{0}\left(f_{j}\right)}+\operatorname{div} X_{0}\left(f_{j}^{\prime}\right) i_{X_{0}\left(f_{j}\right)}\right)
$$

where $f_{j}^{\prime}$ is the orthonormal basis of $n_{+}$corresponding to the orthonormal basis $f_{j}$ of $n_{-} \cdot i_{X}$. denotes the interior product by a vector field $X$. The sum is in fact finite if $\tau_{\Xi}$ belongs to $\Lambda_{H}[\Xi]$. We take $\operatorname{div} X_{n}\left(e_{i}\right)$. for $n<0$ in (2.25), because we consider complexified $L^{2}$ Hilbert space. 
This motivate the following definition:

DefinitionII.2: We call regularized Dirac-Taubes operator over the quotient of the loop group the operator

$$
D_{r, \infty}=\bar{\partial}_{r, \infty}+\bar{\partial}_{r, \infty}^{*}
$$

It is defined over $\Lambda_{H}[\Xi]$, symmetric and therefore closable over $E_{H}[\Xi]$.

Let us compute the fixed point set under the circle action of $L G / H$. It is the only place in this work where it is important to suppose that $H$ is a torus.

Let $g$. be a representative of an element of the fixed point set. We have, for all $s$ :

$$
g_{s+t}=g_{s} k_{t}
$$

for $k_{t}$ in $H$ and

$$
g_{s+t+t^{\prime}}=g_{s+t} k_{t^{\prime}}=g_{s} k_{t} k_{t^{\prime}}=g_{s} k_{t+t^{\prime}}
$$

It follows that $k_{t}=\exp [e t]$ for $e$ belonging to the Lie algebra of $H$. We identify $g . \exp [e$.$] and g^{\prime} \exp \left[e^{\prime}\right.$. $]$ we get for a given element $h^{\prime}$ of $H$ :

$$
g \exp [e t]=g^{\prime} \exp \left[e^{\prime} t\right] k
$$

Therefore, $g=g^{\prime} k$, and since $H$ is commutatif, $e=e^{\prime}$. Moreover, since we consider loops, $e$ must belong to the canonical lattice $L$ of $\mathrm{LieH}$ of $e$ such that $\exp [e]=1$

We have proved:

Theorem II.3: the fixed point set of $L G / H$ under the natural circle action is $G / H \times L$.

\section{LIMIT THEOREMS}

We consider over the group the equation:

$$
d g_{\epsilon, s}=g_{\epsilon, s}\left(\epsilon d B_{s}\right)
$$

starting from $g$ where $B_{s}$ is a Brownian motion over the the Lie algebra of $G$.

It has an heat kernel $p_{s, \epsilon}\left(g, g^{\prime}\right)$. Moreover, $p_{1, \epsilon}(g, g)=p_{1, \epsilon}(e, e)$ clearly. Over the loop group, we consider the probability measure $d \mu_{G, \epsilon}=d \pi g \otimes d P_{\epsilon}(g) . P_{\epsilon}(g)$ is the law of the diffusion $g_{\epsilon, \text {. }}$ starting from $g$ constrained to come back at $g$. We consider a new Hilbert structure for vector fields of the type $X_{s}=g_{s} K_{s}$. It is given by:

$$
\|X .\|_{g_{.}, \epsilon}^{2}=\int_{0}^{1}\left(\left|K_{s}\right|+\epsilon^{-2}\left|d / d s K_{s}\right|^{2}\right) d s
$$

where $K$. is a finite energy application from the circle into the Lie algebra of $H$. This Hilbert structure is invariant under rotation.

An orthogonal basis of the tangent space is given by

$$
X_{n, \epsilon}\left(e_{i}\right)_{s}=g_{\epsilon, s} \frac{\exp [2 i \pi n s] e_{i}}{\sqrt{C n^{2} \epsilon^{-2}+1}}
$$

where $e_{i}$ is an orthonormal basis of the orthogonal of the Lie algebra of $G$. where $e_{i}$ is an orthogonal basis of the Lie algebra of $H$. 
$X_{n, \epsilon}^{j}\left(e^{i}\right)$. are invariant under the $H$ action. Let us compute the divergence of a vector field $X_{n, \epsilon}^{1}\left(e_{i}\right)$. for $\mu_{G, \epsilon}$. For that, we consider $g_{\epsilon, s}^{\lambda}=\exp \left[\lambda \frac{\cos [2 \pi n s] e_{i}}{\sqrt{c n^{2} \epsilon^{-2}+1}}\right]$. We consider the equation (3.1) starting from $e . g_{\epsilon, .}^{\lambda} g_{\epsilon, .}$ is the solution of a differential equation starting from $g_{\epsilon, 0}^{\lambda}$ :

$$
d\left(g_{\epsilon, s} g_{\epsilon, s}^{\lambda}\right)=\left(g_{\epsilon} d g_{\epsilon, s}^{\lambda}\right)+g_{\epsilon, s}^{\lambda}\left(\epsilon d B_{s}\right) g_{s, \epsilon}
$$

.It follows that the law of $g_{\epsilon, s}^{\lambda} g_{\epsilon, .}^{\lambda}$ is equivalent to the original law, if we don't fix the starting point and the end point, that is if we consider the free path group, because the Haar measure is invariant under rotation and because $g_{\epsilon, s}\left(d B_{s}\right) g_{\epsilon, s}$ is still the differential of a Brownian motion on the Lie algebraof $G$. By differentiating in $\lambda=0$, we get infinitisimal integration by parts formulas. By using, the quasi-sure analysis $\left[\mathrm{A}^{\left.-\mathrm{M}_{2}\right]}\right.$, this integration by parts formulas can be desintegrated over the loop group. For cylindrical functionals $F$, we get:

$$
E\left[X_{n, \epsilon}^{1}\left(e_{i}\right) . . F\right]=E\left[F \operatorname{div} X_{n, \epsilon}^{1}\left(e_{i}\right) .\right]
$$

xhere $\operatorname{div} X_{n, \epsilon}^{1}\left(e_{i}\right)$. behaves in small time in $\int_{0}^{1}<\frac{\exp [2 i \pi n s]}{C|n|} e_{i}, d B_{s}>$ in small time because the law og $g_{\epsilon, \text {. }}$ has an equivalent in $g\left(\epsilon B\right.$. $+o\left(\epsilon^{2}\right)$ where $B$. is a flat Brownian bridges over the Lie algebra of $G$. We have supposed that $g_{\epsilon, ;}$ starts from $g$.

So we have the lemma:

Lemma III.1: In law, when $\epsilon \rightarrow 0$, we have over the loop group:

$$
\operatorname{div} X_{n, \epsilon}\left(e_{i}\right) . \rightarrow \int_{0}^{1}<\frac{\exp [2 i \pi n s]}{C|n|} e_{i}, d B_{s}>
$$

where $B_{s}$ is a flat Brownian bridge over the orthogonal of the Lie algebra of $G$.

Let us remark that if we consider the vector field $X_{0 \epsilon}\left(e_{i}\right) .=g_{\epsilon, .} e_{i}$, its divergence is equal to 0 , because the probability law $P_{\epsilon}(g)$ is equal to the probability law of $P_{\epsilon}\left(g g^{\prime}\right)$ by the transformation $g . \rightarrow g . g^{\prime}$.

This leads to the introduction of a limit limit model, according Taubes.

Let us recall that $G$ is endowed with the Haar measure $d \pi($.$) . G / H$ is equipped of a Kaehler structure and over $G / H$, there is the complex line bundle $\xi^{\prime}$, which allows to define the spin structure. We consider the projection $p: G \rightarrow G / H$, and the line bundle $p^{*} \xi^{\prime}$, and the bundle $p^{*}(T(G / H))$. We consider the bundle $V_{g}$ of $g e, e$ belonging to the orthogonal of the Lie algebra of Lie H. It is isomorphic to the bundle $p^{*}(T(H \backslash g))$. It is constituted of vector $g e$ orthogonal to vectors $g e_{i}$ where $e_{i}$ belongs to the Lie algebra of $H$. It can be assimilitated to vectors of the shape $e$ where $e$ belongs to the orthogonal of the Lie algebra of $H$. Let us recall that the complexification of the orthogonal of the Lie algebra of $H$ in the Lie algebra of $G$ can be splitt in $n_{+}(g)+n_{-}(g)$ Moreover, this decomposition is invariant under the action of $H$ by inner product. We consider the family of flat Brownian bridges $g_{\text {flat, }}$ in $V$. starting from 0 in $V_{g}$ endowed with the measure $d \pi(g) \otimes d P_{1, g, f l a t}$. It is the same as $g B_{s}^{1}(g)$ where $B_{s}^{1}(g)$ is a Brownian bridge in the orthogonal of LieH. The tangent space is the space $X_{l, t}$ of path in $V_{g}$ with finite energy. As Hilbert norm, we take:

$$
\left\|X_{l, 0}\right\|^{2}+\int_{0}^{1}\left\|d / d s X_{l, t}\right\|^{2} d t=\left\|X_{l, .}\right\|_{1}^{2}
$$

For forms, we take the Hilbert structure:

$$
\int_{0}^{1}\left|X_{l, t}\right|^{2} d t+\int_{0}^{1}\left|d / d t X_{l, t}\right|^{2} d t=\left\|X_{l}\right\|_{2}^{2}
$$

It has the the orthogonal basis $g \int_{0}^{\cdot} \exp [2 i \pi n s] e_{i}=X_{n, l}\left(e_{i}\right)$ if $n \neq 0$ and $g e_{i}=X_{0, l}\left(e_{i}\right)$. if $n=0$ The complexified tangent space, considered as forms, for the Hilbert structure $\|.\|_{2}^{2}$ has an orthonormal basis $\bar{X}_{n, l}\left(e_{i}\right)_{s}=g \frac{\exp [2 i \pi n s]}{\sqrt{C n^{2}+1}} e_{i}$ for $n \neq 0$. This splitts the space of finite energy paths with values in $V_{g}$ in 
$H_{\text {flat },+}+H_{\text {flat },-}$, where $H_{\text {flat },+}$ corresponds to positive $n$ and $H_{\text {flat }-}$ to negative $n$ in the choice of $\bar{X}_{n, l}\left(e_{i}\right)$. We consider the bundle $n_{-}(g)$ over $G$ and we introduce the following limit bundle:

$$
\Xi_{l}=\Lambda\left(H_{\text {flat },-}\right) \otimes \Lambda\left(n_{-}(g)\right) \otimes p^{*} \xi^{\prime}
$$

The two last parts in $\Xi_{l}$ are pullback bundles by the evaluation map $g_{\text {flat.. }} \rightarrow g$ of bundles over $G$.. This bundle inherites a natural $H$-action, by transforming $\bar{X}_{n, l}\left(e_{i}\right)$ into $\bar{X}_{n, l}\left(h^{-1} e_{i} h\right.$ where $h$ is an element of $h$ and by transforming $X_{0}(f)$ into $X_{0}\left(h^{-1} e_{i} h\right)$, and by an immediate action over $p^{*} \xi$

We consider the Bosonic Fock space $B\left(V_{g}\right)$ over $g_{f l a t, .}$ in $V_{g}$ and we consider the bundle over $G$ :

$$
B\left(V_{g}\right) \otimes \Xi_{l}
$$

Over this limit supersymmetric Fock bundle, we have a connection, which acts as follow: we take the derivative of $e_{i}$ in $\bar{X}_{n, l}\left(e_{i}\right)$. for the trivial connection of $G$ (Let us recall that $V_{g}$ is isomorphic to $p^{*} T((G / H)$ ), which is equal to the pullback of the canonical connection over $\xi^{\prime}$, and which acts over the Wick polynomial $:<e_{i} \alpha_{i} g, g_{f l a t, .}>$ : by taking the derivative of $e_{i}$ and of $g$ in the following formula, but not of $d B^{1}(g)$. Namely for $\alpha_{s}$ a smooth path, we have

$$
<g e_{i} \alpha_{.}, g_{\text {flat }, .}>=\int_{0}^{1}<g e_{i} \alpha_{s}, d g_{f l a t, s}>=\int_{0}^{1}<g e_{i} \alpha_{s}, g d B_{s}^{1}(g)>=\int_{0}^{1}<e_{i}, d B_{s}(g)>
$$

This induces a connection over the bundle over $G B\left(V_{g}\right) \otimes \Xi_{l}$. This bundle inherites clearly an $H$-action, which is compatible with the connection.

We will construct a core wher a "limit" Dirac operator will act. Let us denote this core $\Lambda_{H}\left(\Xi_{l}\right)$. We consider the set of finite combination of $:<g e_{i}^{\prime} \alpha_{i}, g_{\text {flat, }}>: \otimes \bar{X}_{-n, l}\left(e_{i}\right) . \wedge \bar{X}_{0, l}\left(f_{j}^{\prime}\right) . \otimes \psi$ where $e_{i}$ depend only on $g\left(e_{i} g\right.$ belongs in fact to $\left.V_{g}\right), f_{j}^{\prime}$ depends only on $g$, the section $\psi$ on $p^{*} \xi^{\prime}$ depends only on $g$. We consider a finite wedge product of forms $\bar{X}_{-n, l}\left(e_{i}\right)$, with a different convention of sign from $(2.21)$, because these expressions are considered as forms and not as vectors. The same remarks works for $\bar{X}_{0, l}\left(f_{j}^{\prime}\right)$. We remark that each of the elements of $\Lambda_{H}\left(\Xi_{l}\right)$ is not invariant under the $H$-action, in order to get a section of $\Xi_{l}$ which is invariant under the $H$-action, we average it under the $H$-action.

We consider as orthogonal basis of the path with values in $V_{g}$ of finite energy the set constituted of paths $\int_{0}^{t} \exp [2 i \pi n s] e_{i} g d s=X_{n, l}\left(e_{i}\right)_{t}$ and $X_{0, l}\left(f_{j}\right)=f_{j}$ and $X_{0}\left(f_{j}^{\prime}\right)_{=} g f_{j}^{\prime}$.

The infinite dimensional part of the limit Dirac operator is built of the following operator and its adjoint. If we consider a local orthonormal basis $e_{i}$ of $V_{g}$ which depends only on $g$, we have:

$$
\bar{\partial}_{\infty, l}=\sum_{i, n>0} c(n) \nabla_{X_{n, l}\left(e_{i}\right) .} \wedge \bar{X}_{-n, l}\left(e_{i}\right) .
$$

for a family of deterministics constants $c(n)$ for a family of deterministic constant satisfying $\sum c(n)^{2}<\infty$ and we take the sum over an orthogonal basis of the orthogonal of the Lier algebra of $H$. This operator is clearly invariant under the $H$-action, because the set of $h e_{i} h^{-1}$ is still an orthogonal basis of the orthogonal of the Lie algebra of $H$. It acts over $\Lambda_{H}\left(\Xi_{l}\right)$. Namely, if $n \neq 0, X_{n, l}\left(e_{i}\right) .<g e_{j} \alpha, g_{\text {flat }, .}>=\int_{0}^{1}<$ $g e_{j} \alpha_{s}, g \exp [2 i \pi n s] e_{i} d s>$, if $e_{i}$ belongs to the orthogonal of $L i e H$, which is quickly decreasing because $\alpha_{s}$ is chosen smooth. This means that the infinitesimal variation of $d B_{s}$ under $X_{n, l}\left(e_{i}\right)$. is $\exp [2 i \pi n s] e_{i} d s$ if $e_{i}$ belongs to the orthogonal of the lie algebra of $H$.

The adjoint of $\bar{\partial}_{\infty, l}$, denoted by $\bar{\partial}_{\infty, l}^{*}$ is defined by the following formula:

$$
\bar{\partial}_{\infty, l}^{*}=\sum_{i, n<0} i_{X_{-n, l}\left(e_{i}\right)} c(n)\left(-\nabla_{X_{n, l}\left(e_{i}\right) .}+\operatorname{div} X_{n, l}\left(e_{i}\right) .\right)
$$

We put

$$
D_{\infty, l}=\bar{\partial}_{\infty, l}+\bar{\partial}_{\infty, l}^{*}
$$


This operator is clearly invariant under the $H$-action and defined over $\Lambda_{H}\left(\Xi_{l}\right)$. Namely, a vector field $X_{n, l}\left(e_{i}\right)$. is transformed under the $H$ - action into $X_{n, l}\left(h e_{i} h^{-1}\right)$.

The finite dimensional part of the limit Dirac operator is constructed as follow. We add a finite dimensional $\bar{\partial}_{f, l}$ operator:

$$
\bar{\partial}_{f, l}=\sum_{f_{j}} X_{0, l}\left(f_{j}\right) \wedge \nabla_{X_{0}\left(f_{j}\right)}
$$

with the notations of (2.20), which operates over the bundle $B\left(V_{g}\right) \otimes \Xi_{l}$.

We compute its adjoint: with the notations of (2.26), we have:

$$
\bar{\partial}_{f, l}^{*}=\sum_{j}-\nabla_{X_{0}\left(f_{j}^{\prime}\right)} i_{X_{0}\left(f_{j}\right) .}+\operatorname{div} X_{0}\left(f_{j}^{\prime}\right) \cdot i_{X_{0}\left(f_{j}\right)}
$$

In order to summarize us, they are two parts in the limit Dirac operator:

$$
D_{l}=\bar{\partial}_{f, l}+\bar{\partial}_{f, l}+\bar{\partial}_{\infty, l}+\bar{\partial}_{\infty, l}^{*}
$$

There is a finite dimensional part which acts over the bundle $B\left(V_{g}\right) \otimes \Xi_{l}$ and and infinite dimensional part which acts over the Brownian bridge paths in the fiber of the linear bundle $V_{g}$.

The limit model inherites a natural limit circle action. The for $\bar{X}_{n, l}\left(e_{i}\right)$. behaves as $q^{n}$ as we have seen after (2.20).

Let us compute the behaviour under the limit action of the circle of the limit functional $\int_{0}^{1}<g e_{i} \exp [2 i \pi n s], d g_{f l a t, s}>$ for $e_{i}$ a section of $V_{g}$ which depends only on $g$. For that, let us study the action of the circle of the functional $\frac{\int_{0}^{1}<g_{\epsilon, s} e_{i} \exp [2 i \pi n s] d s, d g_{\epsilon, s}>}{\epsilon}$ which tends in law to $\int_{0}^{1}<g e_{i} \exp [2 i \pi n s], d g_{f l a t, s}>$. But

$$
\int_{0}^{1}<g_{\epsilon, s-t} e_{i} \exp [2 i \pi n(s-t)], d g_{s-t, \epsilon}>=\int_{0}^{1}<g_{\epsilon, s} e_{i} \exp [2 i \pi n s], d g_{\epsilon, s}>
$$

If we put $F\left(g_{\epsilon, .}\right)=\int_{0}^{1}<g_{\epsilon, s} e_{i} \exp [2 i \pi n s], d g_{\epsilon, s}>$, we have then

$$
F\left(q g_{\epsilon, .}\right)=q^{n} F\left(g_{\epsilon, .}\right)
$$

for an element $q$ of the circle. This means that $F$ behaves as $q^{n}$ by himself under the natural action of the circle over the loop group. Therefore, $\int_{0}^{1}<g e_{i} \exp [2 i \pi n s], d g_{f l a t, s}>$ behaves as $q^{n}$ by himself under the limit circle action which arises by limit of the circle action over the loop group.

Let us compute the behaviour of this functional under the $H$-action: $g_{\epsilon, s} \exp [2 i \pi n s] e_{i}$ is transformed in $g_{\epsilon, s} \exp [2 i \pi n s] e_{i} h$ and $g_{\epsilon, s}$ is transformed into $g_{\epsilon, s} h$. This shows that the functional $F$ is invariant under the $H$ action, and therefore that the limit functional is invariant under the $H$-action. The core $\Lambda_{H}\left(\Xi_{l}\right)$ is invariant under the limit circle action.

Let us recall what is the Witten genus of $G / H$ : we consider the Atiyah-Singer genus of $G / H$ which gives the Index of the Dirac operator over $G / H$, because $G / H$ is supposed spin. It is too equal to $\operatorname{Todd}(G / H) \wedge$ $\operatorname{ch}\left(\xi^{\prime}\right)$, where $\operatorname{Todd}(G / H)$ is the Todd genus of the Kaehlerian manifold $G / H$. We consider the symmetric tensor algebra of $T(G / H) \otimes C$, where a tensor product of length $j$ is counted with the power $q^{n j}$ where $q$ is the generic element of $S^{1}$. The Witten genus is given by the following formula:

$$
W i(G / H)=\int_{G / H} \hat{A}(G / H) \wedge c h \otimes_{n>0} S_{q^{n}}(T(G / H) \otimes C)
$$

(See [Wi], [T], [Se], [H.B.J]). It is given by the characterisic series (See [H.B.J], p. 83 modulo some normalizing factors):

$$
Q(x)=\frac{x / 2}{\sinh (x / 2)} \prod_{n>0} \frac{1}{\left(1-q^{n} \exp [x]\right)\left(1-q^{n} \exp [-x]\right)}
$$


It is a formal series in $q$. So the Witten genus has to be seen as a formal series in $q$.

In the sequel, we will suppose that $c(n) \neq 0$ for $n>0$.

The operator $D_{l}$ commutes with the limit circle action over $\Lambda_{H}\left(\Xi_{l}\right)$. We can define its "formal" equivariant index:

$$
\operatorname{Ind}_{q} D_{l}=T r_{K e r D_{l}^{+}} q-\operatorname{Tr}_{K e r D_{l}^{-}} q
$$

$D_{l}^{+}$is the restriction of $D_{l}$ to $\Lambda_{H}^{+}\left(\Xi_{l}\right)$, where $\Lambda_{H}^{+}\left(\Xi_{l}\right)$ is the subset of elements of $\Lambda_{H}\left(\Xi_{l}\right)$ where we choose an even power of $\bar{X}_{-n, l}\left(e_{i}\right)$, and $\Lambda_{H}^{-}\left(\Xi_{l}\right)$ is the subset of $\Lambda_{H}\left(\Xi_{l}\right)$. where we have an odd power of $\bar{X}_{-n, l}\left(e_{i}\right)$.

Theorem III.2: The equivariant Index of the limit Dirac operator over $\Lambda_{H}\left(\Xi_{l}\right)$ is equal to the Witten genus of $G / H$.

Proof: Let us compute $\Delta_{l}=D_{l}^{2}$. The infinite dimensional part and the finite dimensional part of the limit Dirac operator anticommute. Therefore, we have:

$$
\Delta_{l}=\left(\bar{\partial}_{f, l}+\bar{\partial}_{f, l}^{*}\right)^{2}+\left(\bar{\partial}_{\infty, l}+\bar{\partial}_{\infty, l}^{*}\right)^{2}=\Delta_{f, l}+\Delta_{\infty, l}
$$

To the polarization given, is associated a class of holomorphic bosons: they are combinations in the Wick products : $\int_{0}^{1}<g e_{j} \exp \left[2 i \pi n_{j} s\right], d g_{f l a t, s}>$ : where $n_{j}>0$. Let $N_{B, c}($ anti) be the second quantized operator of $c(n)^{2}$ which counts the number of antiholomorphic bosons and let $N_{F, c}$ be the second quantized operator of $c(n)^{2}$ which counts the number of fermions of the type $\bar{X}_{-n, l}\left(e_{i}\right)$ with $n>0$. We have $([\mathrm{Ni}])$ :

$$
\Delta_{\infty, l}=N_{B, c}(\text { anti })+N_{F, c}
$$

Namely in the Gaussian space $B$, we do the infinitesimal transformation by $\exp [2 i \pi n s] e_{i}$ where $e_{i}$ belongs to the orthogonal of $\mathrm{LieH}$. Therefore $\Delta_{\infty, l}$ has a set of eigenvectors, which can be seen following Taubes as a countable set of finite dimensional bundles over $G$. Moreover, $\bar{\partial}_{f, l}+\bar{\partial}_{f, l}^{*}$ transforms a section of each bundle into a section of the same finite dimensional bundle. $D_{l}$ has therefore a self-adjoint extension which is compatible with the self-adjoint extension of $\Delta_{l}$. Moreover, these constructions are compatible with the natural $H$-action over the limit model.

This implies that we can compute the elements which are $H$-invariants of the kernel of $\Delta_{l}$ as follows: we take first the kernel of $\Delta_{\infty, l}$, and the elements which are $H$-invariant under the natural $H$-action over it. This gives a countable set of finite dimensional bundles over $G / H$.

$\bar{\partial}_{f, l}+\bar{\partial}_{f, l}^{*}$ acting over $H$-invariant elements of the kernel of $\Delta_{\infty, l}$ tensorized by $H$-invariant sections of $\Lambda\left(n_{-}(g)\right) \otimes p^{*} \xi^{\prime}$ can be seen as homotopic to the Dirac operator over $G / H$ tensorized by the previous countable sets of these finite dimensional bundles over $G / H$. The kernel of the Bosonic number operator is constitute of the holomorphic bosons since $c(n) \neq 0$, or in others words of linear combinations of Wick products : $\int_{0}^{1}<g e_{j} \exp \left[2 i \pi n_{j} s\right], d g_{f l a t, s}>$ : with $n_{j}>0$. This last quantity behaves under the natural circle action as $q^{\sum n_{j}}$. This explains that only positive $n$ appear in the expression of the equivariant Index of $D_{l}$ acting over $\Lambda_{H}\left(\Xi_{l}\right)$. The Index formula (See $[\mathrm{Gi}]$ ) gives:

$$
\operatorname{Ind}_{q}\left(D_{l}\right)=\int_{G / H} \hat{A}(G / H) \wedge \operatorname{ch} \otimes_{n>0} S_{q^{n}}(T(G / H) \otimes C)
$$

$\diamond$

Remark: if we don't suppose that $G / H$ is spin, we get for any complex auxiliary line bundle $\xi$, the equivariant index formula:

$$
\operatorname{Ind}_{q} D_{l}=\int_{G / H} \operatorname{Todd}(H \backslash G) \wedge \operatorname{ch} \xi^{\prime} \wedge \operatorname{ch} \otimes_{n>0} S_{q^{n}}(T(G / H) \otimes C)
$$

Let $B\left(x_{i} ; \delta_{i}\right)$ be a finite cover of $G / H$, such that for all $i$, there exists a small ball $B_{i}$ included in $B\left(x_{i} ; \delta_{i}\right)$ such that the intersection of $B_{i}$ with $B\left(x_{j} ; \delta_{j}\right)$ is empty for $j \neq i$. We consider the open subset $p^{-1} B\left(x_{i} ; \delta_{i}\right)$ of $G$. It constitutes a finite open cover of $G$. 
Let $g$. be a loop. We consider a smooth function $f_{i}$ from $R^{+} \rightarrow[0, \infty]$ which behaves has $\frac{1}{\left(-\delta_{i}{ }^{\prime \prime}+r\right)^{+n}}$ for a big integer $n$ when $r \rightarrow \delta{ }{ }_{i,+}$, and which is infinite if $r<\delta{ }_{i}$ for a convenient $\delta "{ }_{i}$, and which is equal to zero outside a bounded set. We consider the functional

$$
G_{i}^{\prime}(g .)=\int_{H} d \pi(h) \int_{0}^{1} f_{i}\left(d\left(g_{s}, h g_{i}\right) d s\right.
$$

for some $g_{i} \in p^{-1}\left(x_{i}\right)$. We multiply this by a functional of the type (2.16) such that it is equal to infinite over a small neighborhood for the uniform norm of $p^{-1} B\left(x_{i} ; \delta_{i}\right)$ and it is equal to 0 outside a small neighborhood of the same set for the uniform distance in the Holderian loop space. Let us call the functional which is got by this procedure $G_{i}(g$.$) . Let us introduce a function h$ from $[0, \infty] \rightarrow[0,1]$ which is equal to 1 if $r>C$ and which is equal to zero in a neighborhood of 0 .

We introduce the functional:

$$
F\left(g_{.}\right)=h\left(\sum G_{i}(g .)\right)
$$

This functional is clearly invariant by rotation, invariant under the $H$-action. It checks clearly the hypothesis (2.15) and is equal to outside a neighborhood of the constants loops in $G$, and is equal to 1 in a neighborhood of the constant loops in $G$.

Moreover

$$
P\left[F\left(g_{\epsilon, .}\right) \neq 1\right] \leq C \exp \left[-\frac{C}{\epsilon^{2}}\right]
$$

by the exponential inequality.

We put

$$
F_{i}(g .)=h\left(G_{i}\left(g_{.}\right)\right)
$$

Let us suppose that $F_{i}(g.) \neq 0 . \quad g$. lies in a small neighborhood for the uniform topology over the Hoelderian loop space of $p^{-1} B\left(x_{i} ; \delta_{i}\right)$, if $\delta{ }^{\prime}{ }_{i}$ is chosen enough well. This neighborhood is invariant under the $H$-action and under the natural circle action over the loop group. Let $\tau_{i, \xi_{\infty}}(g$.) be the section of the bundle $\xi_{\infty}$ given by the trivialization which is given after $(2.12)$ by 1 . Since the connection form is invariant by rotation, $\tau_{i, \xi_{\infty}}(g$.$) is invariant under rotation. It is too invariant under the H$-action. Namely the connection form (2.14) is invariant under the $H$-action. Let us precise a bit this statement: we consider the set $B(g ; \delta ")$ where $g \in p^{-1}\left(x_{i}\right)$ for the uniform distance over the Hoelderian loop space. We introduce following (2.12) the path in the loop space:

$$
l_{g}(g .)(t)_{s}=\exp \left[t\left(g_{s}-g\right)\right]
$$

joining the loop $g$. to the constant loop $g$, and after we go to $g$ to any fixed given element $g^{\prime}$ of $p^{-1}\left(x_{i}\right)$. If we multiply $g$. by $h$, we consider the ball $B(g h ; \delta ")$ for the uniform distance over the Hoelderian loop space, and the path $l_{h g}(h g.)(t)_{s}=\exp \left[t\left(h g_{s}-h g\right)\right]$ and any given curve in $p^{-1}\left(x_{i}\right)$ joining $g h$ to the given element $g^{\prime}$ of $p^{-1}\left(x_{i}\right)$. Since $-p^{*} R$ is in fact a basical form over $H \backslash G$, the extra integral from curves in $p^{-1} x_{i}$ which appears in the transition functional gives a contribution which is equal to 0 . This explains why the section $\tau_{i, \xi_{\infty}}(g$.$) is invariant under the H$-action.

We consider a section $\tau_{\xi_{\infty}}$ of $\xi_{\infty}$ such that:

$$
\sup _{\epsilon \leq 1} \sum_{n, i} E\left[\left|\nabla_{X_{n}\left(e_{i}\right) .} \tau_{\xi_{\infty}}\left(g_{\epsilon, .}\right)\right|^{2 m}\right]<\infty
$$

It is possible by a small refinment of lemma II.1 to find such a section. By using an argument similar to $\left[\mathrm{L}_{7}\right]$, second remark after Theorem II.10, it is possible to show that these section are dense in the space of $L^{2}$ section of $\xi_{\infty}$ if $\epsilon$ is small enough.

Let us introduce a set of expressions. 
We consider as functionals

$$
\xi(A)=\prod \int_{0}^{1}<g_{\epsilon, s} e_{i} \exp [2 i \pi n s], \delta g_{\epsilon, s}>
$$

where $e_{i}$ is a fixed element of $\operatorname{Lie}(G)$ and $i$ and $n$ describes the finite set $A$ and $\delta$ denotes the Itô integral.

We consider as form $X_{-n}\left(e_{i}\right)$. for $e_{i}$ element of an orthogonal basis of the orthogogonal of the Lie algebra of $H, i$ and the negative integers $-n$ describing the finite set $B$. We consider $X_{n}\left(e_{i}\right)$. for $e_{i}$ belonging to the Lie algebra of $G$ and the negative integers describing a finite set $C$. We consider the wedge product of all and we get an expression called $X_{-}(B, C)$. The different of conventions of signs with (2.20) result that here we consider forms.

At a last step, we consider forms $X_{0}\left(f_{i}^{\prime}\right)$ for $f_{i}^{\prime}$ describing a finite set $C$ of $f_{i}^{\prime}$ (Let us recall that in (2.20), $X_{0}\left(f_{j}\right)$ considered as vector corresponds to $X_{0}\left(f_{j}^{\prime}\right)$ considered as a form). We choose a wedge product of these forms and we call it $X_{0}(D$.$) .$

We consider as core $\Lambda_{H, \epsilon}(\Xi)$ the space of section of $\Xi$ defined by:

$$
\begin{aligned}
& \tau_{\Xi}\left(g_{\epsilon, .}\right)=\sum \lambda(A, B, C, D, i) F_{i}\left(g_{\epsilon, .}\right) \xi(A, i) X_{-}(B, C, i) . \wedge X_{0}(D, i) . \otimes \tau_{i, \xi_{\infty}}\left(g_{\epsilon, .}\right) \\
& \quad+\left(1-F\left(g_{\epsilon, .}\right)\right) \sum F_{I, J, K}\left(g_{\epsilon, .}\right)\left(X_{I .}\right) \wedge Y_{J, .} \otimes \tau_{\xi_{\infty}, K}
\end{aligned}
$$

$\lambda(A, B, C, D, i)$ are constants, where the second sum in (3.34) is defined as in $(2.20)$ and $\tau_{\xi_{\infty}}\left(g_{\epsilon, .}\right)$ satisfies to $(3.33) . F_{I, J, K}\left(g_{\epsilon, .}\right)$ is a cylindrical functional.

This core is invariant under the $H$-action and not under the natural circle action over the loop group. Namely it is not clear that $X_{n, \epsilon}\left(e_{i}\right)$. is invariant under rotation. For that, we average under the circle

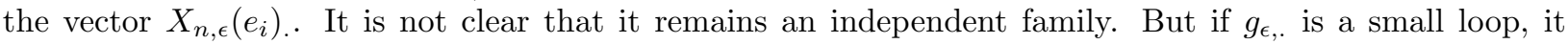
remains an independent family. For that, we introduce some cuttofs as it was done in [J.L] p. 270. If $\sup _{s, t}\left|g_{\epsilon, s} g_{\epsilon, t}^{-1}-e\right|$ remains small, the averaged vector fields are still an independent family of vectors. We introduce the mollifer of [J.L] p.270, which is invariant by rotation and invariant under the $H$ action because $\sup _{s, t}\left|g_{\epsilon, s} g_{\epsilon, t}^{-1}-e\right|$ is invariant under the $H$-action in order to conclude. We localize the core $\Lambda_{H, \epsilon}(\Xi)$ by using this others restrictions, and by using the average vector fields which are invariant by rotations, and which are independent, and which are supposed orthogonal by modifying a bit the metric.

We define Bismut's dilatation $B_{\epsilon}$ which acts over $\xi(A)$ by

$$
B_{\epsilon} \xi(A)=\prod \int_{0}^{1} \frac{<g_{\epsilon, s} e_{i} \exp [2 i \pi n s], d g_{\epsilon, s}>d t}{\epsilon^{\alpha_{i}}}
$$

where $\alpha_{i}=1$ if $e_{i}$ belongs to the orthogonal of the Lie algebra oh $H$ and $\alpha_{i}=1 / 2$ if $e_{i}$ belongs to the Lie algebra of $H$. Bismut's dilatation acts over wedge products $X_{-}(C, D)$ by multiplying each elements of the wedge product of the type $X_{n}\left(e_{i}\right)$ with $e_{i}$ belonging to the Lie algebra of $H$ by $\epsilon$.

Definition III.3: Bismut's dilatation over $\Lambda_{H, \epsilon}(\Xi)$ is defined by:

$$
\begin{gathered}
B_{\epsilon} \tau_{\Xi}\left(g_{\epsilon, .}\right)=\sum F_{i}\left(g_{\epsilon, .}\right) \lambda(A, B, C, D, i) B_{\epsilon} \xi(A, I) B_{\epsilon}\left(X_{-}(B, C, i)\right) \wedge X_{0}(D, i) \otimes \tau_{i, \xi_{\infty}}\left(g_{\epsilon, .}\right) \\
\left(1-F(g . \epsilon, .) \sum F_{I, J, K}\left(X_{I .}\right) \wedge Y_{J} \otimes \tau_{\xi_{\infty}, K}\right.
\end{gathered}
$$

Lemma III.4: Bismut's dilatation are well defined over $\Lambda_{H, \epsilon}(\Xi)$.

Proof: We suppose in order to simplify that $\epsilon=1$.

Since there is a small neighborhood $O_{i}$ of $p^{-1} x_{i}$ for the uniform distance for the Hoelderian loop space such that the intersection of $O_{i}$ with the set $\left\{F_{j}\left(g_{\epsilon, .}\right) \neq 0\right\}$ is empty, the result will follow of the following property: if

$$
i_{O_{i}} \sum_{A, B, C, D} \lambda(A, B, C, D, i) \xi(A, i) X_{-}(B, C, i) \wedge X_{0}(D, i) \otimes \tau_{i, \xi_{\infty}}\left(g_{\epsilon, .}\right)=0
$$

then $\lambda(A, B, C, D, i)=0$. 
We can find a mollifer functional $\tilde{F}_{i}$ which belongs to all the Sobolev spaces and of the type studied before such that $\tilde{F}_{i}(g) \neq 0$ for some $g \in p^{-1} x_{i}$ and such that the set $\left\{\tilde{F}_{i}(g) \neq 0.\right\}$ is included in $O_{i}$.

Let us introduce the random variables $\zeta(i, n)=\int_{0}^{1}<e_{i} \cos [2 \pi n s], d B_{s}>$ for $n \geq 0$ and $\zeta(i, n)=\int_{0}^{1}<$ $e_{i} \sin [2 \pi n s], d B_{s}>$ for $n<0$.

We suppose in order tp simplify the exposure that the diffusion $g$. starts from $e$. We consider the measure over $G \times R^{N} \mu$ :

$$
F \rightarrow E\left[\tilde{F}_{i}(g .) f\left(g_{1}, \zeta(., .)\right]\right.
$$

where we consider $|n| \leq 0$. We can suppose that $\tilde{F}_{i}(e) \neq 0$. Let us show that $\mu$ has an absolutely continuous part which has a strictly positive density in $(e, \zeta)$ for some convenient $\zeta$. This will show the result. It is enough to show the following property: let us replace formally $d B_{s}$ by $h_{s} d s$ with the $L^{2}$ topology over $h . g_{1}$ is replaced by $g_{1}(h$.$) and \zeta(.,$.$) by \zeta(.,).(h$.$) . The property says that the application \Psi: h . \rightarrow\left(g_{1}(h),. \zeta(.,).(h)\right.$. is a submersion fro a small $h$. such that $g_{1}(h)=$.$e . By the positivity theorem of [BA.L] (See [A.K.S] for an$ abstract version), the density part of $\mu$ in $(e, \zeta(.,).(h$.$) is strictly positive.$

If we don't suppose that $n$ can be equal to zero, it is clearly a submersion in $h$. $=0$. The only problem in order that $\Psi$ is a submersion in $h=0$ is $n=0$. But, we can perturb a little bit $h$. in order to get a submersion, because there are multiple iterated integrals which appear in the expression of $g_{1}(h$.). Let us precise a little bit this statement, after plungging the Lie group in an orthogonal matrix group. Let us denote by $D g_{s}(h).\left(h^{\prime}\right)$ the expression of the derivative of $g_{s}\left(h\right.$.) in the direction $h^{\prime}$. It is the solution of the differential equation:

$$
d D g_{s}\left(h_{.}\right)\left(h^{\prime}\right)=D g_{s}\left(h_{.}\right)\left(h_{.}^{\prime}\right) h_{s} d s+g_{s}(h .) h_{s}^{\prime} d s
$$

We can solve this equation by the method of variation of constants. We get:

$$
D g_{1}\left(h_{.}\right)\left(h_{.}^{\prime}\right)=\int_{0}^{1} g_{s}\left(h_{.}\right) h_{s}^{\prime} g_{s}^{-1}(h .) d s g_{1}\left(h_{.}\right)
$$

It is enough to choose a small $h$ such that $s \rightarrow g_{s}(h.) \cdot g_{s}^{-1}(h$.) is not proportional to $s \rightarrow \cos [2 \pi n s]$ and to $s \rightarrow \sin [2 \pi n s]$ for $|n| \leq n_{0}$ such that $g_{1}(h)=e$.

$\diamond$

We would like to compute the limit of $B_{\epsilon} \tau_{\Xi}\left(g_{\epsilon, .}\right)$ when $\epsilon \rightarrow 0$ in order to justify the appellation "limit model" which was used previously.

Definition III.5: we say that a sequence of sections $\tau_{\xi}\left(g_{\epsilon, .}, \epsilon\right)$ of the type

$$
\tau_{\Xi}\left(g_{\epsilon, .}, \epsilon\right)=\sum_{B, C, D, i} F_{I, J, K}\left(g_{\epsilon, .}, \epsilon\right) X_{-}(B, C, i) . \wedge X_{0}(D, i) . \otimes \tau_{\xi_{\infty}, K}\left(g_{\epsilon, 0}\right)
$$

tends in law to the section $\tau_{\Xi_{l}}\left(g_{f l a t, .}\right)$ of the limit model defined by

$$
\tau_{\xi_{l}}\left(g_{f l a t, .}\right)=\sum_{B, C, D, i} \xi_{(B, C, D, i} g_{f l a t, .} \bar{X}_{-, l}(B, C, i) . \wedge \bar{Y}_{0, l}(D, i) . \otimes \tau_{\xi^{\prime}}(g)
$$

if the family of random variables $F_{B, C, D, i}\left(g_{\epsilon, .}, \epsilon\right)$ in $L^{2}(N)$ tends in law to the family of random variable $\xi_{B, C, D, i}\left(g_{\text {flat }, .}\right)$ and if $E\left[\left.\sum F_{I, J, K}\left(g_{\epsilon, .}, \epsilon\right)\right|^{2}\right]$ remains bounded.

In this definition, it is supposed that the random variables $F_{A, B, C, i}\left(g_{\epsilon, .}, \epsilon\right)$ tends in law to zero if there is in $X_{-}(B, C, i)$. a $X_{n}\left(e_{i}\right)$. considered as vector or a $\bar{X}_{-n, l}\left(e_{i}\right)$. considered as a form at the limit (with $\left.n>0\right)$ associated to an element $e_{i}$ of the Lie algebra of $H$.

Proposition III.6: If $\tau_{\Xi}\left(g_{\epsilon, .}\right)$ is a section of $\Xi$ of the type $(3.34), B_{\epsilon} \tau_{\Xi}\left(g_{\epsilon, .}\right)$ tends in law to a section of the limit model $\tau_{\Xi_{l}}$.

Proof:It will result from the following observation.

The contribution which is normalized at which comes from the loop close of the constant loops can be handleded by the following constatation : in law, when $\epsilon \rightarrow 0$

$$
g_{\epsilon, .}=g\left(\epsilon B_{s}+o\left(\epsilon^{2}\right)\right)
$$


for a Brownian bridge $B$. over the Lie algebra of $G$.

This shows that, in law:

$$
B_{\epsilon} \xi(A) \rightarrow \prod_{i, n \in A} \int_{0}^{1}<g e_{i} \exp [2 i \pi n s], d g_{f l a t, s}>
$$

if all the $e_{i}$ belong to the orthogonal of the Lie algebra of $H$ and tends to 0 in law in the others cases. $\diamond$

We consider the core $\Lambda_{H, \epsilon}(\Xi)$ as the set of sections in (3.34) where the contributions which are far from the constant loops are equal to 0 . So this core is a space of sections over a small neighborhood of the constant loops in the loop group which is invariant under the circle action and invariant under the $H$-action. Since the contribution outside the constant loops vanishes, it is natural introduce a deformation of the infinite dimensional Dirac operator near the constant loops.

We define the regularized Dirac-Taubes operator over the core $\Lambda_{H, \epsilon}(\Xi)$ by:

$$
D_{\epsilon}=\bar{\partial}_{\epsilon, \infty}+\bar{\partial}_{\epsilon, \infty}^{*}
$$

where

$$
\bar{\partial}_{\epsilon, \infty}=\sum_{n>0, i} X_{-n}\left(e_{i}\right) . \wedge \nabla_{X_{n, \epsilon}\left(e_{i}\right) .}+\sum_{f_{j}} X_{0}\left(f_{j}^{\prime}\right) \wedge \nabla_{X_{0}\left(f_{j}\right)} .
$$

and where

$$
\bar{\partial}_{\epsilon, \infty}^{*}=\sum_{n<0, i}-\nabla_{X_{n, \epsilon}\left(e_{i}\right) .} i_{X_{n}\left(e_{i}\right) .}+\operatorname{div} X_{n, \epsilon}\left(e_{i}\right) . i_{X_{n}\left(e_{i}\right) .}+\sum_{j}-\nabla_{X_{0}\left(f_{j}^{\prime}\right) .} i_{X_{0}\left(f_{j}\right) .}+\operatorname{div} X_{0}\left(f_{j}^{\prime}\right) . i_{X_{0}\left(f_{j}\right)} .
$$

Let us remark that $D_{\epsilon}$ operates over the core $\Lambda_{H, \epsilon}(\Xi)$. Namely:

$$
\begin{aligned}
& X_{m, \epsilon}\left(e_{j}\right) \cdot \int_{0}^{1}<g_{\epsilon, s} \exp [2 i \pi n s] e_{i}, d g_{\epsilon, s}>=O(1 / n) \\
& +C(\epsilon, m) \int_{0}^{1}<g_{\epsilon, s} \exp [2 i \pi n s] e_{i}, g_{\epsilon, s} \exp [2 i \pi m s] e_{j}>=O(1 / n)+C(\epsilon, m, n) \delta_{m, n}
\end{aligned}
$$

The problem is that this operator is not invariant under rotation. For that, we average the vector fields $X_{n, \epsilon}\left(e_{i}\right)$. under the natural circle action. The problem is that the averaged vector fields do not constitute an independent family in general. But it remains an independent family, because we work over a small neighborhood of the constant loops. We have the following theorem:

THEOREM III. 7: when $\epsilon \rightarrow 0$, we have in law if $\tau_{\Xi}\left(g_{\epsilon, .}\right)$ is a section belonging to $\Lambda_{H, \epsilon}^{\prime}(\Xi)$

$$
D_{\epsilon} B_{\epsilon} \tau_{\Xi}\left(g_{\epsilon, .}\right) \rightarrow D_{l} \tau_{\Xi_{l}}
$$

for some $c(n) \neq 0, \tau_{\Xi}\left(g_{\epsilon, .}\right)$ and $\tau_{\Xi_{l}}$ being given as in Proposition III.6.

\section{Proof:}

We remark first that the contribution of the derivatives of $F_{i}\left(g_{\epsilon .}\right)$ vanishes as it was seen previously.

There is the contribution of the covariant $\tau_{i, \xi_{\infty}}\left(g_{\epsilon, .}\right)$. If $n \neq 0, \nabla_{X_{n, e_{i}}\left(e_{i}\right)} \tau_{i, \xi_{\infty}}\left(g_{\epsilon, .}\right)$ tends clearly to 0 because $X_{n, \epsilon}\left(e_{i}\right) \rightarrow 0$. If $n=0$, we remark that $\xi_{\infty}$ resticts over $G$ to $p^{*} \xi^{\prime}$, and the connection forms over the first bundle over the loop group restricts over the connection form over $G$.. Let us look at formula (2.15). The distinguished path $l_{g^{\prime}}\left(\epsilon_{\epsilon,}\right)(t)$ tends to the distinguished path joining $g$ to the given element $g^{\prime}$ of $p^{-1}\left(x_{i}\right)$. Therefore

$$
A_{g^{\prime}}\left(g_{\epsilon,}\right)\left(X_{0}\left(e_{i}\right) .\right) \rightarrow A_{g^{\prime}}\left(e_{i} g\right)
$$

where $A_{i}$ is the connection form associated to $p^{*} \xi^{\prime}$ over $p^{-1} B\left(x_{i} ; \delta\right)$. Therefore the expression which comes to the covariant derivative of $\tau_{i, \xi_{\infty}}\left(g_{\epsilon, .}\right)$ pass to the limit. 
The treatment of the covariant derivative of $B_{\epsilon}\left(X_{-}(B, C, i)\right.$.) and $X_{0}(D, i)$. is trivial, with the chosen connections.

The treatment of $\operatorname{div} X_{n, \epsilon}\left(e_{i}\right)$. is given at Lemma III.1. It remains to remark that $\operatorname{div} X_{n, \epsilon}^{2}\left(e_{i}\right)$. at the limit does not appear, because there is an interior product in $D_{\epsilon}, i_{X_{n}\left(e_{i}\right)}$, and that the corresponding forms are multiplied by $\epsilon$ and disappear at the limit.

It remains to consider

$$
X_{n, \epsilon}\left(e_{i}\right) . B_{\epsilon} \xi(A)
$$

or equivalently:

$$
X_{n, \epsilon}\left(e_{i}\right) \cdot \int_{0}^{1}<g_{\epsilon, s} \exp [2 i \pi m s] e_{j}, \delta g_{\epsilon, s}>/ \epsilon
$$

Let us consider first $n \neq 0$ and $e_{j}$ belonging to the orthogonal of $L i e H$. It tends in law too

$$
\int_{0}^{1}<g \exp [2 i \pi n s] e_{j}, g \exp [2 i \pi m s] e_{i}>
$$

which is the derivative of $\int_{0}^{1}<\operatorname{gexp}[2 i \pi m s] e_{j}, d g_{f l a t, s}>$ along $X_{n, l}\left(e_{i}\right)$.

If $n \neq 0$ and $e_{j}$ belongs to $\operatorname{LieH}, X_{n, \epsilon}\left(e_{i}\right) .=O(\epsilon)$ and

$$
\left.X_{n, \epsilon}\left(e_{i}\right) \cdot \int_{0}^{1}<g_{\epsilon, s} \exp [2 i \pi m s] e_{j}, \delta g_{\epsilon, s}>/ \sqrt{\epsilon}=O\left(\epsilon^{1 / 2}\right)\right)
$$

and tends to zero, which is the derivative of the limit of this expression, which is 0 .

If $n=0$ and if $e_{j}$ belongs to the orthogonal of the Lie algebra of $H$,

$$
\begin{gathered}
X_{0}\left(e_{i}\right) \cdot \int_{0}^{1}<g_{\epsilon, s} \exp [2 i \pi m s] e_{j}, \delta g_{\epsilon, s}>/ \epsilon \\
=\int_{0}^{1}<g_{\epsilon, s} e_{i} \exp [2 i \pi m s] e_{j}, \delta g_{\epsilon, s}>/ \epsilon+\int_{0}^{1}<g_{\epsilon, s} \exp [2 i \pi m s] e_{j}, \delta g_{\epsilon, s}\left(e_{i}\right)>/ \epsilon
\end{gathered}
$$

which tends in law to

$$
\int_{0}^{1}<g e_{i} \exp [2 i \pi m s d] e_{j}, g d B_{s}>+\int_{0}^{1}<g \exp [2 i \pi m s] e_{j}, g e_{i} d B_{s}>=0
$$

which is the derivative of $\int_{0}^{1}<\exp [2 i \pi m s] e_{j}, d g_{f l a t, s}>=\int_{0}^{1}<\exp [2 i \pi m s] e_{j}, d B$. $>$ in the direction $g e_{i}$.

We have consider the limit in law of each component of the section $\tau_{\Xi}\left(g_{\epsilon, .}\right)$, but the collections of each components converges in law.

The last property of proposition III.5. is clearly checked.

Remark: It is enough to supposed $G / H$ spin, by extending the Clifford multiplication over small loops as it was done in [J.L] (2.10), because we work over a small neighborhood of the constant loops.

Remark: The previous argument should justify, but not proove the following assertion: over $L(G) / H$, there is a Dirac operator, and its "renormalized" equivariant index is equal to the Witten genus of $G / H$. The contribution of the others fixed points should be treated by looking at the "homeomorphism" $g$. $\rightarrow \exp [e]$.$g .$

\section{REFERENCES}

[Ai] Aida S.: Sobolev Spaces over loop groups. J.F.A. 127 (1995), 155-172. 
[A.K.S] Aida S. Kusuoka S. Stoock D.: On the support of Wiener functionals. In " Asymptotics Problems in Probability theory: Wiener functionals and asymptotics". Elworthy K.D. Ikeda N. edit. Longman Scientific 284 (1993), 3-35.

[A.M $\left.{ }_{1}\right]$ Airault H. Malliavin P.: Integration on loop groups. Publication Paris VI. (1991).

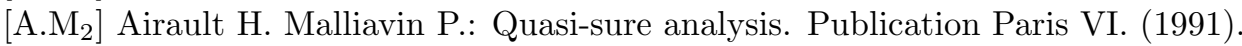

[A.H-K] Albévério S. Hoegh-Krohn R.: The energy representation of Sobolev Lie groups. Compositio Math. 36 (1978), 37-52.

[Ar] Arai A.: A path integral representation of the Index of Kaehler-Dirac operators on an infinite dimensional space. J.F.A. 82 (1989), 330-369.

[At] Atiyah M.: Circular symmetry and stationary phase approximation. Conference in honour of L. Schwartz. Astérisque 131 (1985), 43-59.

[BA.L] Ben Arous G. Léandre R.: Décroissance exponentielle du noyau de la chaleur sur la diagonale. II. P.T.R.F. 90 (1991), 377-402.

[B.V] Berline N. Vergne M.: Zéros d'un champ de vecteur et classes caractéristiques équivariantes. Duke Math. Journal. 50 (1983), 539-548.

$\left[\mathrm{Bi}_{1}\right]$ Bismut J.M.: Large deviations and the Malliavin Calculus. Progress in Math. 45. Birkhauser. (1984).

$\left[\mathrm{Bi}_{1}\right]$ Bismut J.M.: The Lefschetz fixed point formulas. J.F.A. 57 (1984), 329-348.

$\left[\mathrm{Bi}_{2}\right]$ Bismut J.M.: Index theorem and equivariant cohomology on the loop space. C.M.P. 98 (1985), 213-237.

$\left[\mathrm{Bi}_{3}\right]$ Bismut J.M.: Localisation formulas, superconnections and the index theorem for families. C.M.P. 103 (1986), 127-166.

[Br] Brylinski J.L.: Loop spaces, characteristic classes and geometric quantization. Progress in Maths. 107. Birkhauser. (1992).

[C.M] Carey A.L. Murray M.K.: String structure and the path fibration of a group. C.M.P. 141 (1991), 441-452.

[C.P] Coquereaux R. Pilch K.: String structures on loop bundles. C.M.P. 120 (1989), 353-378.

[D.H.V.W] Dixon L. Harvey J. Vafa C. Witten E.: Strings on orbifold. Nuclear. Phys. B 261 (1985), 678-686.

[F.Fr] Fang S. Franchi J.: De Rham-Hodge-Kodaira operator on loop groups. J.F.A. 148 (1997), 391-407.

[Gi] Gilkey P.B.: Invariance theory, the heat equation and the Atiyah-Singer theorem. Publish and Perish. (1984).

[Gr 1 ] Gross L.: Logarithmic Sobolev inequalities on loop groups. J.F.A. 102 (1991), 268-313.

$\left[\mathrm{Gr}_{2}\right]$ Gross L.: Uniqueness of ground states for Schrodinger operators over loop groups. J.F.A. 112 (1993), $373-441$.

[H.B.J] Hirzebruch F. Berger T. Jung R.: Manifolds and modular forms. Aspect of mathematics 20. Vieweg (1993).

[H] Hirzebruch F.: Elliptic genera genera of level $N$ for complex manifolds. In "Differential geometrical methods in theoretical physics" Bleuler K. Werner M. edit Kluwer (1988), 37-63.

[L.L.W] Jaffe A. Lesniewski A. Weitsman J.: Index of a family of Dirac operators on loop space. C.M.P. 112 (1987), $75-88$.

[J.L] Jones J.D.S. Léandre R.: A stochastic approach to the Dirac operator over the free loop space. In " Loop spaces and groups of diffeomorphisms". Proceedings of the Steklov Institute 217 (1997), 253-282.

[K.N] Kobayashi S. Nomizu S.: Foundations of differential geometry . I. Interscience (1969).

[K] Kusuoka S.: More recent theory of Malliavin Calculus. Sugaku 5.2. (1992), 155-173.

$\left[\mathrm{L}_{1}\right]$ Léandre R.: Intégration dans la fibre associée a une diffusion dégénérée. P.T.R.F. 76. (1987), 341-358.

$\left[\mathrm{L}_{2}\right]$ Léandre R.: Applications quantitatives et qualitatives du Calcul de Malliavin. In "French-Japanese Seminar". M. Métivier S. Watanabe edit. L.N.M. 1322 (1988), 109-133. English translation. In "Geometry of random motion". R. Durrett M. Pinsky edit. Contemporary Maths. 73 (1988), 173-197.

$\left[\mathrm{L}_{3}\right]$ Léandre R.: Brownian motion over a Kaehler manifold and elliptic genera of level N. In "Stochastic analysis and applications in Physics". Sénéor R. Streit L. edit. Nato Asie Series 449. (1994), 193-219.

$\left[\mathrm{L}_{4}\right]$ Léandre R.: Hilbert space of spinor fields over the free loop space. Reviews in Math. Physics. 9.2. (1997), 243-277.

$\left[\mathrm{L}_{5}\right]$ Léandre R.: Stochastic gauge transform of the string bundle. J. Geometry and Physics. 26 (1998), 1-25.

$\left[\mathrm{L}_{6}\right]$ Léandre R.: String structure over the Brownian bridge. J. Maths. Physics. 40.1 (1999), 454-479. 
$\left[\mathrm{L}_{7}\right]$ Léandre R.: Cover of the Brownian bridge and stochastic symplectic action. To be published in Reviews in Math. Physics.

$\left[\mathrm{L}_{8}\right]$ Léandre R.: Stochastic cohomology of Chen-Souriau and line bundle over the Brownian bridge. To be published in P.T.R.F.

[L.R] Léandre R. Roan S.S.: A stochastic approach to the Euler-Poincaré number of the loop space of a developable orbifold. J. Geometry. and Physics. 16 (1995), 71-98.

[M.L] Mac Laughlin D.: Orientation and string structures on loop spaces. Pac. J. Math. 155 (1992), 143-156.

[Ma] Malliavin P.: Stochastic Analysis. Springer. (1997).

[N] Nishimura T.: Exterior bundle over a complex abstract Wiener space. Preprint (1990).

[Se] Segal G.: Elliptic cohomology. Séminaire Bourbaki 695. Astérisque 161-162 (1988), 187-201.

[Sh ${ }_{1}$ ] Shigekawa I.: Transformations of Brownian motion on a Riemannian symmetric space. Z. W. 65 (1984), 493-522.

$\left[\mathrm{Sh}_{2}\right]$ Shigekawa I.: Differential Calculus on a based loop group. In "New Trends in Stochastic Analysis". K.D. Elworthy S. Kusuoka I. Shigekawa edit. World Scientific. (1997), 375-398.

[S.W] Spera M. Wurzbacher T.: In preparation.

[T] Taubes C.: $S^{1}$ actions and elliptic genera. C.M.P. 122 (1989), 455-526.

[U] Uglanov T.: Surface integrals and differential equations on an infinite dimensional space. Sov. Math. Dok. 20. 4 (1979), 917-920.

[Wa] Watanabe S.: Stochastic analysis and its applications. Sugaku 5.1. (1992), 51-71.

[Wi] Witten E.: The index of the Dirac operator in loop space. In "Elliptic curves and modular forms in algebraic topology". Landweber P.S. edit. L.N.M. 1326 (1988), 161-181.

Institut Elie Cartan

Département de mathématiques

Université Nancy I

54000. Vandoeuvre-les-Nancy

FRANCE 